Check for updates

Cite this: J. Mater. Chem. C, 2021 , 9, 10768

Received 9th December 2020, Accepted 8th February 2021

DOI: $10.1039 / \mathrm{d0tc05773h}$

rsc.li/materials-c

\title{
Switching of the mechanism of charge transport induced by phase transitions in tunnel junctions with large biomolecular cages $\dagger$
}

\author{
Nipun Kumar Gupta, $\ddagger^{\text {ab }}$ Rupali Reddy Pasula, $\ddagger^{c}$ Senthil Kumar Karuppannan, (D) $\ddagger^{a}$ \\ Zhang Ziyu, ${ }^{a}$ Anton Tadich, ${ }^{d}$ Bruce Cowie, ${ }^{d}$ Dong-Chen Qi, ${ }^{e}$ Peter Bencok, \\ Sierin Lim (D) *cg and Christian A. Nijhuis (iD *abh
}

\begin{abstract}
Tunnel junctions based on Fe storing globular proteins are an interesting class of biomolecular tunnel junctions due to their tunable Fe ion loading, symmetrical structure and thermal stability, and are therefore attractive to study the mechanisms of charge transport (CT) at the molecular level. This paper describes a temperature-induced change in the CT mechanism across junctions with large globular ( $25 \mathrm{~nm}$ in diameter) E2-proteins bioengineered with Fe-binding peptides from ferritin (E2-LFtn) to mineralise Fe ions in the form of iron oxide nanoparticles (NPs) inside the protein's cavity. The iron oxide NPs provide accessible energy states that support high CT rates and shallow activation barriers. Interestingly, the CT mechanism changes abruptly, but reversibly, from incoherent tunnelling (which is thermally activated) to coherent tunnelling (which is activationless) across the E2-LFtn-based tunnel junctions with the highest Fe ion loading at a temperature of 220-240 K. During this transition the current density across the junctions increases by a factor of 13 at an applied voltage of $V=-0.8 \mathrm{~V}$. X-ray absorption spectroscopy indicates that the iron oxide NPs inside the E2-LFtn cages undergo a reversible phase transition; this phase transition opens up new a tunnelling pathway changing the mechanism of CT from thermally activated to activationless tunnelling despite the large size of the E2-LFtn and associated distance for tunnelling.
\end{abstract}

\section{Introduction}

In biological processes including photosynthesis, ${ }^{1,2}$ enzymatic catalysis, ${ }^{3}$ DNA transcription or repair, ${ }^{4,5}$ charge transport (CT)

\footnotetext{
${ }^{a}$ Department of Chemistry, National University of Singapore, 3 Science Drive, Singapore 117543, Singapore

${ }^{b}$ Centre for Advanced $2 D$ Materials, National University of Singapore, 6 Science Drive 2, Singapore 117546, Singapore

${ }^{c}$ School of Chemical and Biomedical Engineering, Nanyang Technological University, 70 Nanyang Drive, Singapore 637457, Singapore

${ }^{d}$ Australian Synchrotron Clayton, Victoria 3168, Australia

${ }^{e}$ Centre for Materials Science, School of Chemistry and Physics, Queensland University of Technology, Brisbane, Queensland 4001, Australia

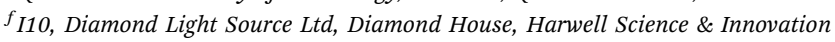
Campus, Didcot, Oxfordshire, OX11 ODE, UK

${ }^{g}$ NTU-Northwestern Institute for Nanomedicine, Nanyang Technological University, 50 Nanyang Avenue, Singapore 639798, Singapore.E-mail: slim@ntu.edu.sg

${ }^{h}$ Hybrid Materials for Opto-Electronics Group, Department of Molecules and Materials, Faculty of Science and Technology, University of Twente,

7500 AE Enschede, The Netherlands. E-mail: c.a.nijhuis@utwente.nl

$\dagger$ Electronic supplementary information (ESI) available. See DOI: 10.1039/ d0tc05773h

\$ These authors contributed equally.
}

is highly efficient in nature, but the reasons why this is so are unclear. One of the key challenges is to identify the conduction pathways across proteins which can be realised by incorporating proteins in tunnel junctions and to study how CT depends, on for example, site-selective modifications of the proteins, ${ }^{6,7}$ mechanical deformation of proteins, ${ }^{8,9}$ orientation of the protein with respect to the electrode, ${ }^{10,11}$ or the role of the cofactors. ${ }^{12-16}$ Specifically, it is challenging to distinguish between the contributions to the mechanism of CT from the protein shell and the co-factors or (multiple) heme groups. ${ }^{712,17-20}$ Cahen and Sheves and co-workers demonstrated that replacement of the native metal ion co-factor with another metal ion changes the mechanism of CT from coherent tunnelling (which is activationless) to incoherent tunnelling (which is thermally activated and also called hopping). ${ }^{12,21}$ The mechanism of CT also changes from coherent to incoherent tunnelling when the metal centre of azurin $(\mathrm{Az}),{ }^{12}$ or the ferrihydrite core of ferritin, ${ }^{15}$ was removed. Here, we report a change in the mechanism of CT as a function of the Fe ion loading and temperature in tunnel junctions with large (diameter of $25 \mathrm{~nm}$ obtained from its crystal structure ${ }^{22}$ ) globular E2 proteins bioengineered with Fe binding sites from ferritin, E2-LFtn, allowing us to the control the iron oxide content inside the cavity ranging from 
500 to $3000 \mathrm{Fe}$ ions per E2-LFtn cage. Depending on the Fe ion loading, the mechanism of CT switches from incoherent to coherent tunnelling caused by a thermally induced phase transition of the iron oxide NPs inside the E2-LFtn. The mechanism of CT across junctions with the E2-LFtn lacking the iron oxide NPs (i.e., apo-E2-LFtn) is incoherent tunnelling. These results give us new insights into the conduction pathways of tunnel junctions with large proteins.

Various mechanisms have been proposed to describe CT across proteins, which can be roughly divided into two classes: coherent tunnelling which is independent of temperature and incoherent tunnelling which depends on temperature. The general tunnelling equation (eqn (1)) shows how fast the tunnelling current density $\left(J\right.$ in $\mathrm{A} \mathrm{cm}^{-2}$ ) decays (determined by the tunnelling decay coefficient $\beta$ in $\mathrm{nm}^{-1}$ ) as a function of the tunnelling barrier width $d$ (in $\mathrm{nm}$ ) where $J_{0}$ (in $\mathrm{A} \mathrm{cm}^{-2}$ ) is the pre-exponential factor.

$$
J=J_{0} e^{-\beta d}
$$

The first-order description of incoherent tunnelling is given by the Arrhenius equation (eqn (2)), where charges hop over an energy barrier with activation energy $\left(E_{\mathrm{a}}\right.$ in $\left.\mathrm{meV}\right)$, where $k_{\mathrm{B}}$ is the Boltzmann constant and $T$ is the temperature (in K).

$$
J=J_{0} \mathrm{e}^{\frac{-E_{\mathrm{a}}}{k_{\mathrm{B}} T}}
$$

Coherent CT with low values of $\beta$ in range of 0.8 to $1.6 \mathrm{~nm}^{-1}$ for photosystem-I (PS-I), ${ }^{10} 1.9$ to $3.3 \mathrm{~nm}^{-1}$ for $\mathrm{Az},{ }^{23} 0.28$ to $1.3 \mathrm{~nm}^{-1}$ for ferritin, ${ }^{15}$ have been reported. These values are much lower than $\beta$ values for aliphatic SAMs $\left(\beta=7-11 \mathrm{~nm}^{-1}\right)^{24,25}$ or aromatic SAMs $\left(\beta=2-5 \mathrm{~nm}^{-1}\right)^{26-28}$ where coherent tunnelling dominates. Normally, such low values of $\beta$ are indicative of incoherent tunnelling, but these examples lack a thermally activated component (eqn (2)) which suggest that CT is coherent in nature. This type of CT is also so-called long-range tunnelling and is still poorly understood. ${ }^{29}$ Possible explanations include flickering resonance, ${ }^{30}$ superexchange, ${ }^{31}$ bias-induced coherent transfer ${ }^{32}$ or sequential incoherent tunnelling with multiple low energy barriers. ${ }^{33,34}$

Incoherent tunnelling has been observed in tunnel junctions with various proteins including bacteriorhodopsin (bR), ${ }^{13}$ cytochrome- $C(\mathrm{Cyt}-C)^{17}$ and in proteins without the co-factor, e.g. , apo-Az, ${ }^{12}$ apo-ferritin ${ }^{15}$ and apo-PS-I ${ }^{10}$ (but the same junctions with the co-factor showed thermally activated CT). Remarkably, for a few systems a change in incoherent tunnelling regime (each characterised by a different value of $E_{\mathrm{a}}$ ) has been observed as a function of $T$ or $d$. For example, in bR junctions where the $\pi$-conjugated retinal co-factor is decoupled from the bR protein (reconstituted bR), the value of $E_{\mathrm{a}}=490 \mathrm{meV}$ at high $T(T=340-260 \mathrm{~K})$ is substantially larger than the value of $E_{\mathrm{a}}=180 \mathrm{meV}$ at low $T(T=260-200 \mathrm{~K}) .{ }^{13}$ Similarly, in Cyt- $C$ junctions with the $\mathrm{Fe}$ ion removed from the porphyrin ring, a low $E_{\mathrm{a}}=105 \mathrm{meV}$ for $T=285-200 \mathrm{~K}$ while a high $E_{\mathrm{a}}=550 \mathrm{meV}$ for $T=335-285 \mathrm{~K}$ was observed. ${ }^{17}$ A transition from coherent to incoherent tunnelling with $d$ has been observed for conjugated molecular wires, ${ }^{28,35}$ but a transition from coherent tunnelling with large $\beta$ to low $\beta$ with increasing $d$ has been reported for junctions with long molecular wires ${ }^{27,28,35}$ or ferritin. ${ }^{15}$ These examples highlight the different types of CT regimes that can be accessed in (bio)molecular tunnel junction although the underlying reasons why the mechanism of CT changes are not fully understood.

The E2 protein is a chemically and thermally stable (up to $85{ }^{\circ} \mathrm{C}$ ) cage-like globular protein which naturally occurs as a part of the pyruvate hydrogenase multi-enzyme complex in the thermophile Geobacillus stearothermophilus and is responsible for
(A)
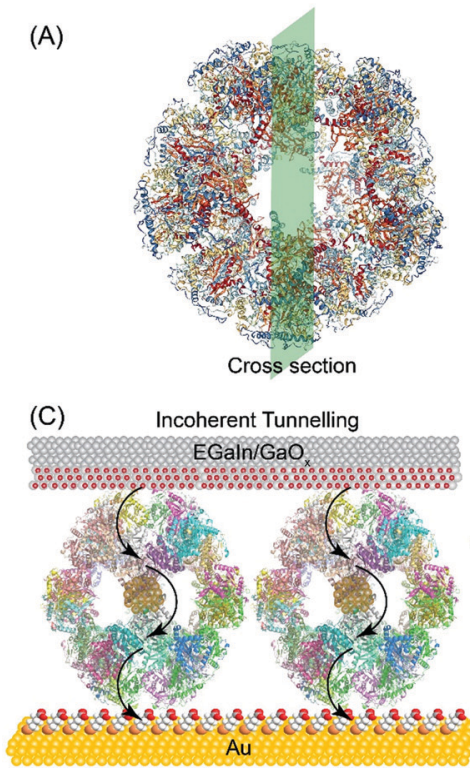

(B)
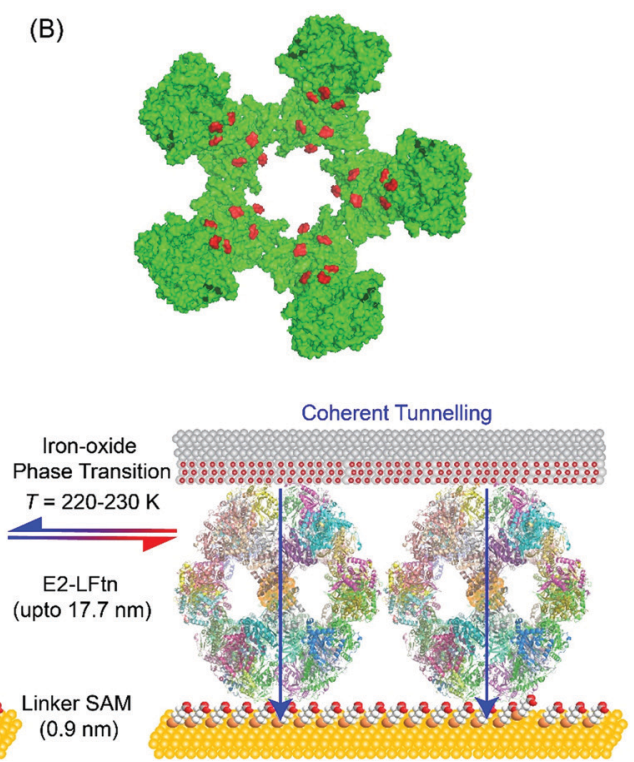

Fig. 1 (A) PyMOL representation of apo-E2-LFtn and (B) cross-section where the Fe ion binding sites are highlighted in red colour. (C) Schematic illustration of the Au-linker-E2-LFtn//GaO $/$ EGaln tunnel junction. Here, "//" represents a van der Waals contact, "/" represents the contact between the $\mathrm{GaO}_{x}$ and EGaln, "-" represents a chemical contact, the black arrows represent incoherent tunnelling, and the blue arrow represents coherent tunnelling. 
linking glycolysis and the Krebs cycle. ${ }^{36}$ The E2 protein consists of 60 identical subunits self-assembled into a hollow spherical architecture with an external diameter of $24 \mathrm{~nm}$ and an internal diameter of $12 \mathrm{~nm}$ (Fig. 1). The cage structure has 12 openings, each with a diameter of $5 \mathrm{~nm}$. The E2 protein has been bioengineered with frog M-ferritin mimicking peptides to form E2-LFtn, ${ }^{22}$ which can mineralise $\mathrm{Fe}^{\mathrm{II}}$ into iron oxide NPs in a similar fashion to ferritin. ${ }^{15,37,38}$ The structure, magnetic behaviour and composition of the iron oxide NPs in ferritin is a function of the Fe ion loading/cage and its mineralisation conditions have been studied in detail. The E2-LFtn stores the Fe ions as iron oxide NPs (3-6 nm in size) in its hollow core. $^{22}$ Amongst the large variety of iron oxides known, $\mathrm{Fe}^{\mathrm{II}}$ always exists in an octahedral $\left(O_{\mathrm{h}}\right)$ symmetry state while $\mathrm{Fe}^{\mathrm{III}}$ can exist in both $O_{\mathrm{h}}$ and tetrahedral $\left(T_{\mathrm{d}}\right)$ symmetry states. ${ }^{39,40}$ The Fe ions are stored predominately in the form of ferrihydrite in ferritin, but other iron oxide phases can be present in varying degrees depending on the preparation methods, Fe ion loading and types of ferritin. ${ }^{41,42}$ Iron oxides readily undergo thermally-induced phase transitions. For instance, phase transitions of ferrihydrite to other iron oxide phases (including lepidocrocite $\left(\gamma-\mathrm{Fe}^{\mathrm{III}} \mathrm{OOH}\right)$, goethite $\left(\alpha-\mathrm{Fe}^{\mathrm{III}}\right.$ $\mathrm{OOH}){ }^{43}$ haematite $\left(\alpha-\mathrm{Fe}_{2} \mathrm{O}_{3}\right)^{44}$ and magnetite ${ }^{45}\left(\mathrm{Fe}_{3} \mathrm{O}_{4}\right)$ in NPs have been investigated in detail. We note that it has been reported that $\mathrm{Fe}^{\mathrm{II}}$ ions can assist in phase transitions involving ferrihydrite. ${ }^{43,45-47}$ These examples highlight the complexity of iron oxide chemistry which, as we show here, can lead to interesting CT phenomena across biomolecular junction with E2-LFtn.

We report a thermally induced reversible and irreversible switching of the mechanism of CT across biomolecular tunnel junctions with bioengineered E2-LFtn. The mechanism of CT across apo-E2-LFtn is characterised by two distinct incoherent tunnelling regimes with high $(254 \pm 32 \mathrm{meV})$ and low $(24 \pm 1 \mathrm{meV})$ values of $E_{\text {a }}$, while the mechanism of CT across E2-LFtn is characterised by a single incoherent tunnelling regime with an intermediate $E_{\mathrm{a}}$ (35-61 meV) depending on the iron oxide loading. Thus, we conclude that the iron oxide NPs provide accessible energy states that support an alternate incoherent tunnelling pathway. For junctions with E2-LFtn with a large Fe ion loading (3000Fe), reversible conduction switching was observed at $T=220-240 \mathrm{~K}$ which was accompanied by a change in the CT mechanism from incoherent tunnelling in the low conduction state to coherent tunnelling in the high conduction state. Temperature-dependent X-ray absorption spectroscopy (XAS) indicates a reversible phase transition of the iron oxide NPs inside the E2-LFtn. These results suggest that the mechanism of CT can be switched as a function of temperature and iron oxide loading in E2-LFtn biomolecular tunnel junctions. Our findings give new insights in the conduction pathways of biomolecular junctions defined by the protein cage and the quantity of co-factors present inside the protein and demonstrate how these conduction pathways can be controlled.

\section{Results and discussion}

\section{Design of the junctions}

Fig. 1A shows the PyMOL representation of apo-E2-LFtn, showing the globular shape and the cavity with the structural information obtained from Protein Data Bank with identification number 1B5S. The $\mathrm{Fe}^{\mathrm{II}}$ binding chemistry of the frog M-ferritin mimicking peptides in E2-LFtn has been reported previously. ${ }^{22,37}$ Each of the 60 subunits of the E2 cage has been modified with the catalytic binding sites from frog M-ferritin making it possible to load the E2 cage with iron oxide NPs (see ref. 22 for all details and characterisation of E2-LFtn). The Fe ion content of E2-LFtn can be controlled similar to that reported for controlled loading of ferritin. ${ }^{15,48}$ In this study, we loaded E2-LFtn with 500-3000 Fe ions/cage (at higher loadings, the iron oxide precipitated from solution). Fig. 1B shows the corresponding cross-section of apoE2-LFtn with the Fe binding sites highlighted in red. Fig. 1C illustrates the Au-linker-E2-LFtn//GaO ${ }_{x} /$ EGaIn junction and illustrates the iron oxide phase transition inside the E2-LFtn as a function of temperature, which, in turn, modulates the possible mechanism of CT and induces conductance switching. The preparation and characterisation of apo-E2-LFtn and E2-LFtn loaded with iron oxide NPs are briefly described in Sections S1-S3, ESI $\dagger$ ). Before using E2-LFtn, we characterised the size of the proteins and confirmed that E2-LFtn retained its globular structure after mineralisation with dynamic light scattering technique, as shown in Fig. S1A (ESI $\dagger$ ). The actual Fe ion content present in the E2-LFtn was characterised with inductively coupled plasma atomic emission spectroscopy (see Fig. S1B, ESI $\dagger$ ).

The smooth $\mathrm{Au}$ bottom electrodes were obtained with template-stripping (Section S4, ESI $\dagger$ ), on which we formed the self-assembled monolayers (SAMs) of E2-LFtn using a linker SAM (Section S5, ESI $\dagger$ ). ${ }^{15}$ The linker SAM was derived from 6-mercaptohexanoic acid and was activated with 1-ethyl-3-(3dimethylaminopropyl)carbodiimide (EDC); these activated carboxyl groups react with the $\mathrm{NH}_{2}$ groups located at the periphery of E2-LFtn to form covalent amide bonds ensuring the proteins are firmly anchored to the Au surface. After monolayer formation, the EGaIn top electrode was introduced using cone-shaped EGaIn tips ${ }^{49}$ or with EGaIn stabilised in a microchannel perpendicularly aligned over an array of micropores in $\mathrm{Al}_{2} \mathrm{O}_{3}$ fabricated on $\mathrm{Au} .^{50}$

\section{Characterisation of the E2-LFtn monolayers}

Fig. 2A shows an atomic force microscope (AFM) image of a bare Au substrate (see Section S6 for experimental details, ESI $\dagger$ ) with a root mean square (rms) surface roughness of $0.49 \mathrm{~nm}$ measured over an area of $1.0 \times 1.0 \mu^{2}$, which is similar to previously reported values. ${ }^{49}$ Fig. $2 \mathrm{~B}$ shows an AFM image of a densely packed monolayer of 1000Fe ion loaded E2-LFtn on the $\mathrm{Au}$-linker surface with a rms surface roughness of $2.6 \mathrm{~nm}$ (measured over an area of $1.0 \times 1.0 \mu \mathrm{m}^{2}$ ). The line scans are presented in Fig. S2 (ESI $\dagger$ ). To determine whether E2-LFtn retained its globular structure upon adsorption, we used tapping mode AFM to determine the height of individual proteins from the AFM height profiles recorded for sub-monolayers of E2-LFtn on Au-linker. Fig. 2C shows an example of an AFM image for a sub-monolayer of apo-E2-LFtn where individual proteins can be seen (see Section S6 for additional images, ESI $\dagger$ ), can be seen. We used a similar procedure to obtain AFM height profiles of E2-LFtn as a function of Fe ion loading. Fig. 2D shows the histograms 
(A)

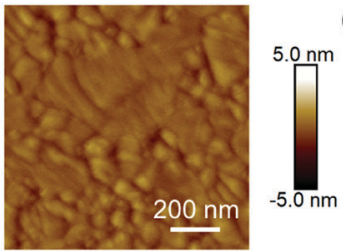

(D)

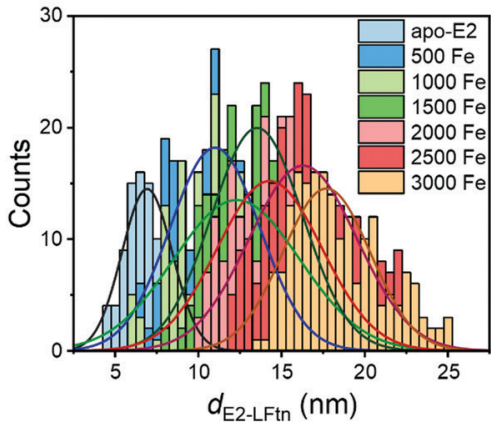

(B)

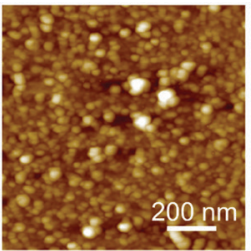

(C)

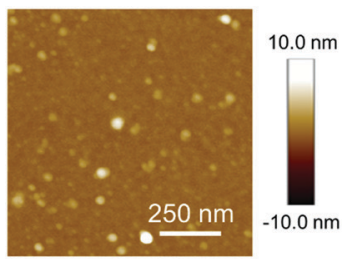

(E)

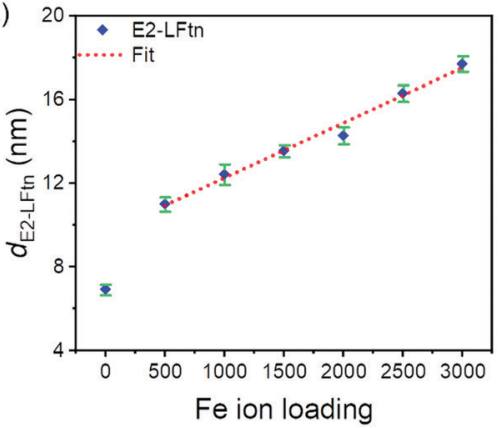

Fig. 2 AFM images of the bare template-stripped Au surface (A), 1000Fe E2-LFtn monolayer on Au (B), and a sub-monolayer of apo-E2-LFtn on Au (C). (D) Histograms of $d_{\mathrm{E} 2-\mathrm{LFtn}}$ as a function of Fe ion loading along with Gaussian fits these histograms. (E) The value of $d_{\mathrm{E} 2 \text {-LFtn }} v s$. Fe ion loading (error bars represent 95\% confidence levels), where the red dashed line indicates the correlation between $d_{\mathrm{E} 2 \text {-LFtn }}$ and the Fe loading $\left(R^{2}=0.985\right)$.

along with Gaussian fits to these histograms of the heights of individual E2-LFtn proteins, $d_{\mathrm{E} 2-\mathrm{LFtn}}$ (in $\mathrm{nm}$ ), as a function of Fe ion loading, and the $d_{\mathrm{E} 2 \text {-LFtn }}$ shows the linear dependence with Fe ion loading (Fig. 2E). The apo-E2-LFtn forms thin layers with a measured height of $6.9 \pm 0.3 \mathrm{~nm}$, which is much smaller than the hydrodynamic diameter of the protein measured in solution, $d_{\mathrm{HD}, \mathrm{E} 2 \text {-LFtn }}=25 \mathrm{~nm}$, from which we conclude that it does not retain it globular structure and likely forms dense, flattened monolayers (since these junctions do not short, see below). In contrast, the E2-LFtn loaded with iron show larger diameter for loading as low as 500Fe E2-LFtn the value of $d_{\mathrm{E} 2 \text {-LFtn }}$ of $11.0 \pm 0.3 \mathrm{~nm}$ indicating that, although the protein flattens, the globular structure is preserved. The highest value of $d_{\mathrm{E} 2-\mathrm{LFtn}}$ of $17.7 \pm 0.4 \mathrm{~nm}$ for maximum Fe ion loading which is close to $d_{\mathrm{HD}}$, E2-LFtn $=25 \mathrm{~nm}$. This observation can be attributed to compression induced by the AFM tip or loss of water from the cavity. ${ }^{51,52}$ We note that these observations are consistent with previously reported thickness values of ferritin Aftn-AA, $d_{\mathrm{Aftn}}$, on the same Aulinker surface as a function of $\mathrm{Fe}$ ion loading. ${ }^{15}$ The ratio between maximum protein height obtained from the AFM image $\left(d_{\mathrm{Aftn}}=10.9 \mathrm{~nm}\right)$ and hydrodynamic radius (obtained from dynamic light scattering measurements for ferritin Aftn-AA $\left.d_{\mathrm{HD}, \mathrm{Aftn}}=12.1 \mathrm{~nm}\right)$ is $d_{\mathrm{Aftn}} / d_{\mathrm{HD}, \mathrm{Aftn}}=0.90$, which is closer to unity than that of E2-LFtn of $d_{\mathrm{E} 2-\mathrm{LFtn}} / d_{\mathrm{HD} \text {,E2-LFtn }}=0.70$, from which we conclude that the E2-LFtn is more dynamic and flexible than ferritin Aftn-AA.

\section{Characterisation of iron oxide NPs inside E2-LFtn}

To investigate the composition of the iron oxide NPs inside the E2-LFtn cages immobilised on the Au-linker surface as a function of temperature, we measured the $\mathrm{Fe}_{\mathrm{L}_{2,3}}$ edge XAS (Section $\mathrm{S} 7$, $\mathrm{ESI} \dagger)$. This technique resolves the presence of $\mathrm{Fe}^{\mathrm{II}}$ and $\mathrm{Fe}^{\mathrm{III}}$ ions along with their atomistic symmetry states. Fig. 3 shows the $\mathrm{Fe} \mathrm{L}_{3}$ edge XAS spectra of the monolayers of 3000 and 2000Fe E2-LFtn recorded at three different temperatures of
$T=300 \mathrm{~K}$, followed by cooling down to $120 \mathrm{~K}$, and after heating back to $300 \mathrm{~K}$. The peak observed at $707.5 \mathrm{eV}$ is assigned to $\mathrm{Fe}^{\mathrm{II}}$. The peak at $709.2 \mathrm{eV}$ is assigned to $\mathrm{Fe}^{\mathrm{III}}$ and corresponds to $\mathrm{Fe}$ cations in both $O_{\mathrm{h}}$ and $T_{\mathrm{d}}$ symmetries. The ratio (in both $T_{\mathrm{d}}$ and $O_{\mathrm{h}}$ geometries) of $\mathrm{Fe}^{\mathrm{II}}$ and $\mathrm{Fe}^{\mathrm{III}}$ were obtained from modelling of the XAS data with the CTM4XAS software ${ }^{53}$ (see Section S7 for details, ESI $\dagger$ ) and Fig. 3 shows the results. Interestingly, the iron oxide NPs inside E2-LFtn are dominated by $\mathrm{Fe}^{\mathrm{II}}$ rather than $\mathrm{Fe}^{\mathrm{III}}$ ions as reported for ferritin. ${ }^{54,55}$ This observation suggests that the iron mineralisation process in E2-LFtn differs from that of ferritin which could be related to the differences in cavity size and spatial arrangement of the M-ferritin mimicking catalytic sites. ${ }^{22}$ The ratio of the peak areas $\left(R_{\mathrm{I}}=\mathrm{Fe}^{\mathrm{II}} / \mathrm{Fe}^{\mathrm{III}}\right)$ for 3000Fe E2-LFtn decreases with decreasing $T$ from 1.25 at $300 \mathrm{~K}$ to 0.50 at $120 \mathrm{~K}$ (Fig. $3 \mathrm{~A}, \mathrm{~B}$ and Table 1 ). On heating back to $300 \mathrm{~K}$, Fig. 3C shows that the $R_{\mathrm{I}}$ ratio increases to 1.00 which is nearly its original value indicating that this phase transition is reversible. In contrast, for 2000Fe E2-LFtn, initially no signal could be recorded at $300 \mathrm{~K}$ (Fig. 3D) but a signal at the $\mathrm{Fe}_{3}$ edge was recovered at $300 \mathrm{~K}\left(R_{\mathrm{I}}=5.00\right.$, Fig. $\left.3 \mathrm{~F}\right)$ after cooling to $120 \mathrm{~K}\left(R_{\mathrm{I}}=2.00\right.$, Fig. $\left.3 \mathrm{E}\right)$. This observation can be explained as follows. Initially, the iron oxide NPs are buried in the protein cavity hampering their detection, but (partial) denaturation of the protein induced by cooling to low $T$ exposes the iron oxide nanoparticles and, consequently, increases the XAS signals. Denaturation also explains the irreversible nature of the corresponding junction (discussed below). We also note that $3000 \mathrm{Fe}$ E2-LFtn has a higher Fe ion content than 2000Fe E2-LFtn, which is apparently high enough to conduct XAS measurements around the $\mathrm{Fe} \mathrm{L}_{3}$ edge despite the iron oxide nanoparticles being inside the protein cavity and thus shielded by a $\sim 6 \mathrm{~nm}$ thick protein shell. We confirmed the presence of iron oxide at $300 \mathrm{~K}$ for intact protein using a beam line with a larger beam flux (Section S8, ESI $\dagger$ ). These results suggest that Fe ion loading stabilises the E2-LFtn which agrees with the larger 
(A) 3000Fe E2-LFtn
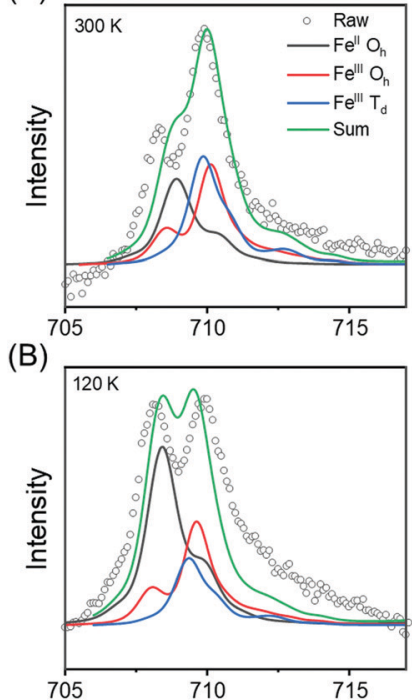

(D) 2000Fe E2-LFtn

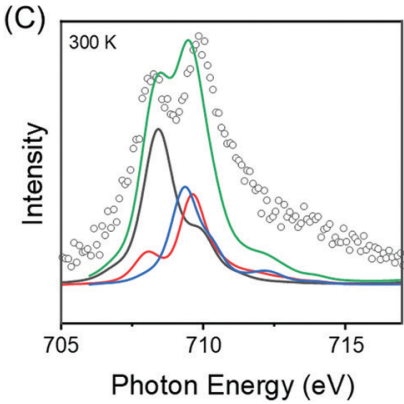

(E)
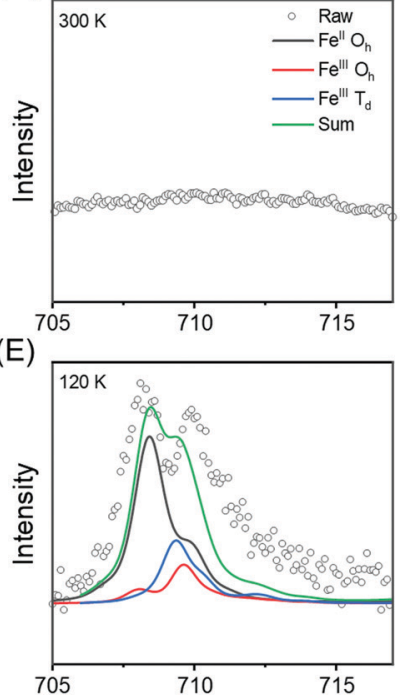

(F)

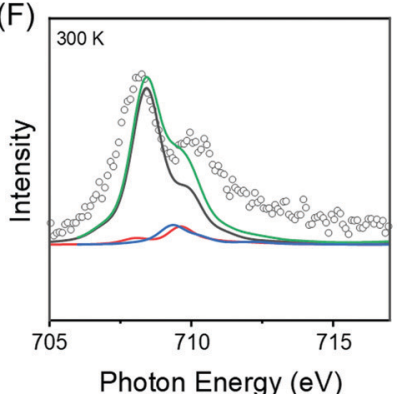

Fig. 3 XAS data around the $\mathrm{Fe}_{3}$ edge recorded from a monolayer of $3000 \mathrm{Fe}$ E2-LFtn on Au at $300 \mathrm{~K}(\mathrm{~A}), 120 \mathrm{~K}$ (B) and after heating back to $300 \mathrm{~K}(\mathrm{C})$, and the same but for a monolayer of 2000Fe E2-LFtn $300 \mathrm{~K}(\mathrm{D})$, $120 \mathrm{~K}(\mathrm{E})$ and after heating to $300 \mathrm{~K}(\mathrm{~F})$. The solid lines are fits as explained in the text.

Table 1 Peak intensity ratio of $2000 \mathrm{Fe}$ and $3000 \mathrm{Fe}$ E2-LFtn as a function of temperature

\begin{tabular}{llll}
\hline Sample & $R_{\mathrm{I}}$ at $300 \mathrm{~K}($ initial) & $R_{\mathrm{I}}$ at $120 \mathrm{~K}$ & $R_{\mathrm{I}}$ at $300 \mathrm{~K}$ (final) \\
\hline $2000 \mathrm{Fe}$ & - & 2.00 & 5.00 \\
$3000 \mathrm{Fe}$ & 1.25 & 0.50 & 1.00
\end{tabular}

value of $d_{\text {E2-LFtn }}=17.7 \pm 0.4 \mathrm{~nm}$ for $3000 \mathrm{Fe}$ E2-LFtn than $d_{\text {E2-LFtn }}=16.3 \pm 0.4 \mathrm{~nm}$ for $2000 \mathrm{Fe}$ E2-LFtn indicating that flattening of $2000 \mathrm{Fe}$ E2-LFtn is more significant than for $3000 \mathrm{Fe}$ E2-LFtn. We note that Rakshit et al. ${ }^{52}$ reported an increase in the Young's modulus of ferritin as a function of $\mathrm{Fe}$ ion loading. From these results we conclude that 3000Fe E2-LFtn forms stable monolayers supporting a reversible phase transition of the iron oxide NPs inside the E2-LFtn cavity.

\section{Charge transport measurements}

To investigate the mechanism of CT across E2-LFtn, we formed electrical contacts to the SAMs of E2-LFtn with cone-shaped tips of EGaIn (Fig. 1C) and measured the $J(V)$ characteristics as a function of Fe ion loading. We followed previously reported

procedures for the junction formation, data collection and statistical data analysis (see Section S8 for details, ESI $\dagger$ ). ${ }^{49}$ We recorded 400-440 $J(\mathrm{~V})$ curves for each type of junction and plotted all values of $\log _{10}|J|$ for each measured applied bias voltage in histograms to determine the Gaussian average value of $\log _{10}|J|,\left\langle\log _{10}|J|\right\rangle_{\mathrm{G}}$, and the Gaussian log-standard deviation, $\sigma_{\log }$. Fig. 4A shows the $\left\langle\log _{10}|J|\right\rangle_{\mathrm{G}} v s . V$ curves as a function of the Fe ion loading and Fig. 4B shows the corresponding current decay plot at $-0.5 \mathrm{~V}$. The value of $J$ exponentially decays with the value of $d_{\mathrm{E} 2-\mathrm{LFtn}}$ and fitting the data to eqn (1) yields $\beta=0.39 \pm 0.02 \mathrm{~nm}^{-1}$. It is noteworthy that the CT distance is at $11.0 \mathrm{~nm}$ for $500 \mathrm{Fe}$ E2-LFtn, and nearly $17.7 \mathrm{~nm}$ for $3000 \mathrm{Fe}$ E2-LFtn meaning these proteins are highly conductive (see below). We note that the values of $J$ for junctions with apo-E2-LFtn are similar to the junctions of $500 \mathrm{Fe}$ and $1000 \mathrm{Fe}$ E2-LFtn. This observation can be explained by the notion that the shorter $d_{\mathrm{E} 2-\mathrm{LFtn}}$ of the apo-E2-LFtn junction $(6.9 \mathrm{~nm} v s$. 11-12 nm for junctions with $500 \mathrm{Fe}$ or $1000 \mathrm{Fe}$ E2-LFtn) compensates for the lack of tunnelling sites associated with the iron oxide particles.

\section{Temperature-dependent CT}

To investigate the mechanism of CT in more detail, we performed $J(V, T)$ measurements for the E2-LFtn junctions. The proteins were immobilised on $\mathrm{Au}$ (following the same procedure as described above) inside micropores $\mathrm{AlO}_{x}$ over which microchannels filled with EGaIn were aligned following previously reported methods, and the fabrication and experimental details are presented in Sections S9 and S10 (ESI $\dagger) .{ }^{50}$ Fig. 4C shows the Arrhenius plots at $V=-0.5 \mathrm{~V}$ for junctions with monolayers of E2-LFtn with $1500 \mathrm{Fe}, 2000 \mathrm{Fe}$ and $3000 \mathrm{Fe}$ loading along with fits to
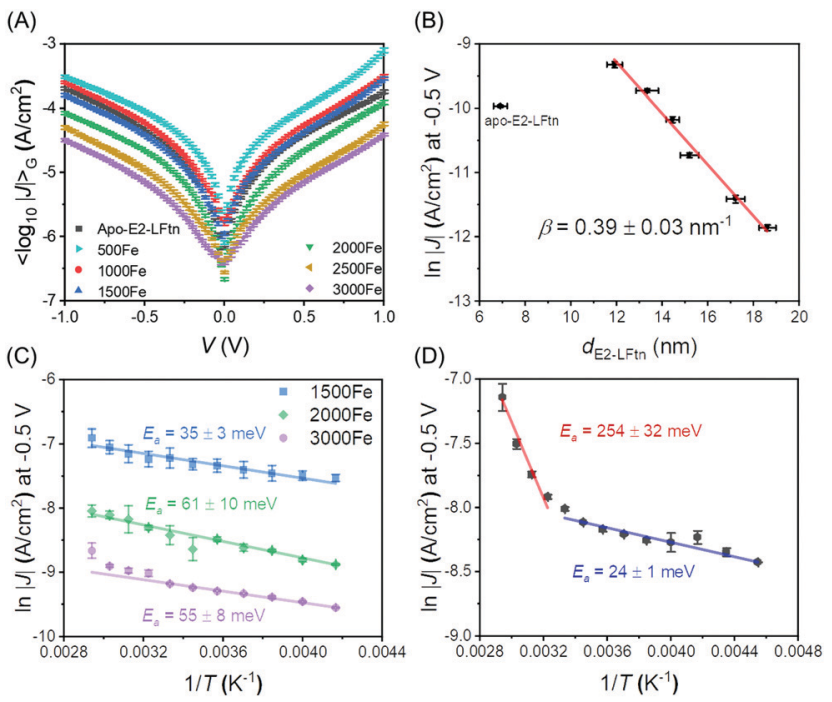

Fig. 4 Plots of $\left\langle\log _{10}|J|\right\rangle_{G} v s . V(A$ and $B)\langle\ln |J|\rangle_{G}$ at $-0.5 V$ vs. $d_{E 2-L F t n}$ (error bars in $J$ and $d_{\mathrm{E} 2 \text {-LFtn }}$ represent the $95 \%$ confidence levels, solid line is a fit to eqn (1)). (C) Arrhenius plots of the values of $J$ measured at $-0.5 \mathrm{~V}$ and $T=240-340 \mathrm{~K}$ for junctions with E2-LFtn with different Fe ion loadings. (D) Arrhenius plot for junctions with apo-E2-LFtn at $-0.5 \mathrm{~V}$ in the range of $T=230-340 \mathrm{~K}$. Error bars represent the standard deviation obtained from 3 different junctions and the solid lines in panels $C$ and $D$ are fits to eqn (2). 
eqn (2) to determine $E_{\mathrm{a}}$. The mechanism of CT is incoherent tunnelling with small values of $E_{\mathrm{a}}$ ranging from $35 \pm 3$ to $61 \pm 10 \mathrm{meV}$. This incoherent tunnelling mechanism explains the small value of $\beta$ (Fig. 4B).

Fig. 4D shows the Arrhenius plots at $V=-0.5 \mathrm{~V}$ for junctions with apo-E2-LFtn along with fits to eqn (2). Remarkably, two distinct incoherent tunnelling regimes can be observed with a value of $E_{\mathrm{a}}$ of $254 \pm 32 \mathrm{meV}$ for $T=310-340 \mathrm{~K}$ and $E_{\mathrm{a}}=24 \pm 1 \mathrm{meV}$ for $T=230-300 \mathrm{~K}$. The transition temperature, $T_{\mathrm{T}}$, is around $300 \mathrm{~K}$. A similar transition $\left(T_{\mathrm{T}}=290 \mathrm{~K}\right)$ between two incoherent tunnelling regimes $\left(E_{\mathrm{a}}=550 \mathrm{meV}\right.$ to $\left.E_{\mathrm{a}}=105 \mathrm{meV}\right)$ has been reported for junctions with apo-Cyt- $C$ (where the Fe ion was removed from the porphyrin). ${ }^{17}$ In contrast, for junctions with holo-Cyt- $C$ proteins, the Fe ion increased the CT rates by a factor of two and only one incoherent tunnelling regime with $E_{\mathrm{a}}=105 \mathrm{meV}$ dominates CT indicating that the Fe ion opens up more efficient CT pathways. ${ }^{17}$ Thus, our junctions show a similar behaviour where two thermally activated CT pathways are observed for junctions with apo-E2-Lftn. As discussed above in the AFM characterisation section, apo-E2-LFtn immobilised on the surface does not retain its cage-like structure and forms a disordered protein layer where charges must hop between different disorganised protein segments which constitute relatively large incoherent tunnelling barriers. This incoherent tunnelling mode is only active at relatively high temperatures $\left(T>T_{\mathrm{T}}\right)$. In contrast, only one thermally activated CT pathway with relatively low $E_{\mathrm{a}}$ is observed for junctions with holo-E2LFtn where the iron oxide NPs likely provide (multiple) low energy incoherent tunnelling sites. Here, the charges cross the protein segments of an organised monolayer where the globular tertiary structure of the protein is preserved. This difference in structure of the junctions, in conjunction with low energy incoherent tunnelling sites provided by the iron oxide NPs, could explain the large observed $E_{\mathrm{a}}$ for apo-E2-LFtn and low $E_{\mathrm{a}}$ for holo-E2-LFtn. At $T<T_{\mathrm{T}}$, incoherent tunnelling across segments becomes energetically unfavourable and a different pathway dominates CT with low $E_{\mathrm{a}}$. Often, low $E_{\mathrm{a}}$ values are associated with "efficient" incoherent tunnelling, but here the currents at low $T$ are small (since the primary conduction channel at high $T$ is turned off). Therefore, at low $T$ another mechanism is at work where incoherent tunnelling may, for instance, involve nuclear tunnelling, ${ }^{56}$ partial loss of coherence, ${ }^{57}$ or voltage induced changes to electronic states. ${ }^{32}$

\section{Controlled switching of CT}

Fig. 5 shows the switching behaviour of representative junctions with $3000 \mathrm{Fe}$ and 2000Fe E2-LFtn (see Section S11 for additional data sets, ESI $\dagger$ ). As we have reported before, the shape of the $J(V)$ curves recorded with junctions with flat EGaIn stabilised in microchannels are, unlike rough cone-shaped EGaIn tips, not obscured by artefacts caused by the high surface roughness of cone-shape EGaIn tips. ${ }^{50,58}$ Fig. 5A shows the $J(V)$ traces recorded during cooling of the 3000Fe E2-LFtn junction from 340 to $150 \mathrm{~K}$ and Fig. 5B shows the same during warming up back to $340 \mathrm{~K}$. Fig. $5 \mathrm{C}$ shows the values of $J$ at $V=-0.8 \mathrm{~V}$ for the cooling and heating cycles. We observe that the junction undergoes a reversible transition at $220 \mathrm{~K}$ from a low to a high conduction state with 13-fold increase in the $J$. The value of
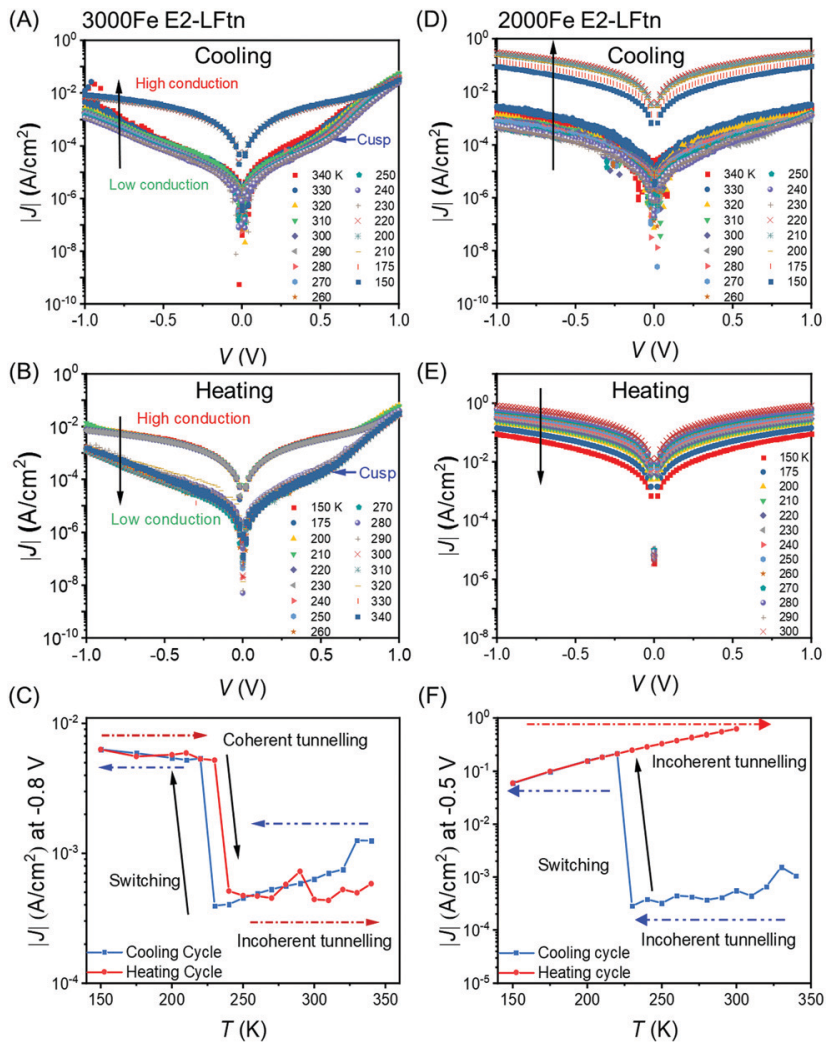

Fig. 5 Temperature-dependent $J(V)$ measurements of cooling (A) and heating of a $3000 \mathrm{Fe}$ E2-LFtn junction, and that of (D-E) 2000 Fe E2-LFtn junction in the range of $T=150-340 \mathrm{~K}$. The temperature dependency of $|\mathrm{J}|$ at $-0.8 \mathrm{~V}$ for $3000 \mathrm{Fe}$ E2-LFtn (C) and 2000Fe E2-LFtn (F) junctions.

$J$ restores to its original value at $240 \mathrm{~K}$ during the heating cycle, but a small hysteretic behaviour is visible. This behaviour coincides with the reversible phase transition of the iron oxide NPs inside 3000Fe E2-LFtn observed with XAS as described above. Therefore, we conclude that the phase transition of the iron oxide NPs modulates the conduction across the E2-LFtn tunnel junctions.

Fig. 5D-F shows the same for a 2000Fe E2-LFtn junction, but here an irreversible transition occurs at $220 \mathrm{~K}$ during cooling with a factor of 40 increase in $J$ at $V=-0.8 \mathrm{~V}$, which is likely result of (partial) protein denaturation of 2000Fe E2-LFtn upon cooling. This behaviour resembles the irreversible phase transition of the iron oxide NPs inside 2000Fe E2-LFtn as observed with XAS as described above (see Fig. 3). Junctions with low Fe ion loading (i.e., 1500Fe E2-LFtn) and apo-E2-LFtn shorted at $T=240-230 \mathrm{~K}$. These observations suggest that the current switching observed in 2000Fe E2-LFtn junctions is caused by partial failure due to denaturation of the protein. Denaturation of 2000Fe E2-LFtn also could explain the temperature variable XAS described above reinforcing this interpretation of the $J(V, T)$ data.

Interestingly, CT across 3000Fe E2-LFtn junctions in the high temperature regime $(T=220-340 \mathrm{~K})$ is thermally activated with $E_{\mathrm{a}}=55 \pm 8 \mathrm{eV}$ at $-0.8 \mathrm{~V}$ during cooling but not in the low temperature regime $(T=150-220 \mathrm{~K}$; Fig. 5C). In contrast, the corresponding $J(V)$ curves recorded from 2000Fe E2-LFtn 


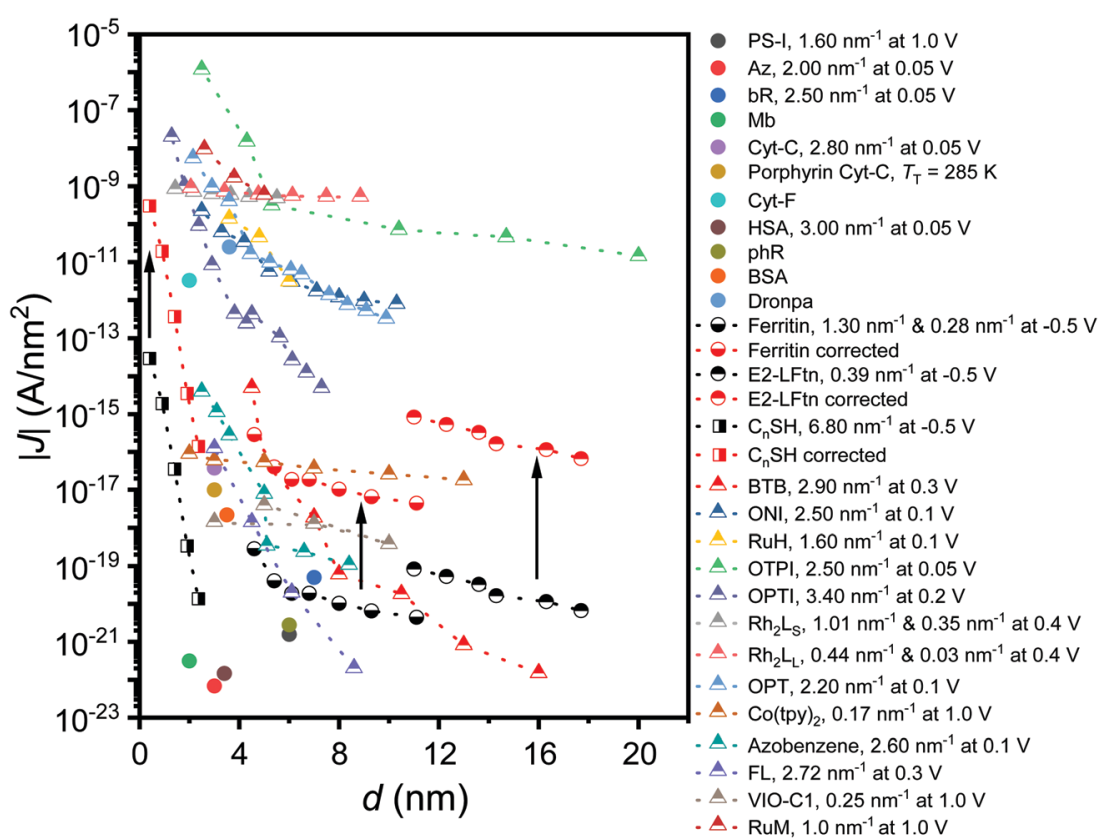

Fig. $6 \mathrm{~J}$ at $0.1 \mathrm{~V}$ as a function of the $d$ for alkanethiols (squares) taken from ref. 60 . The proteins (circles) presented are PS-I, ${ }^{10} \mathrm{bR},{ }^{13,23}$ ferritin, ${ }^{15} \mathrm{Cyt}-\mathrm{C}$ and Cyt- $C$ with Fe ions removed (porphyrin-Cyt-C), ${ }^{17,23}$ halorhodopsin (phR), ${ }^{14} \mathrm{Mb},{ }^{61} \mathrm{Az}$, cytochrome- $\mathrm{F}(\mathrm{Cyt}-\mathrm{F})$, dronpa, bovine serum albumin $(\mathrm{BSA})$ and human serum albumin (HSA). ${ }^{23}$ The conjugated molecular wires (triangles) presented here are bis-thienylbenzene (BTB), ${ }^{28} 1$ - $(4-$-aminophenyl)-1'methyl-4,4'-bipyridinium bis-hexafluorophosphate (VIO-C1), ${ }^{27}$ OPTI, ${ }^{59}$ oligo-tetrathiafulvalene-pyromelliticdiimide-imine (OTPI), ${ }^{62}$ oligonaphthalenefluoreneimine $(\mathrm{ONI}){ }^{63}$ copper bipyridine $\left(\mathrm{Co}(\mathrm{tpy})_{2}\right),{ }^{64}$ oligophenylenetriazole $(\mathrm{OPT}),{ }^{65}$ fluorene $(\mathrm{FL}),{ }^{66} \mathrm{Ru}$-bis $\left(\sigma\right.$-arylacetylide) wires with - $\mathrm{CH} \mathrm{H}_{2}-$ termini (RuM) and $-\mathrm{O}\left(\mathrm{CH}_{2}\right)_{6}$ - termini $(\mathrm{RuH}){ }^{67}$ azobenezene, ${ }^{68}$ and $\left[\mathrm{Rh}_{2}\left(\mathrm{O}_{2} \mathrm{CCH}_{3}\right)_{4}\right]\left(\mathrm{Rh}_{2}\right)$ wires with linear with pyrazine $\left(\mathrm{L}_{S}\right)\left(\mathrm{Rh} 2 \mathrm{~L}_{\mathrm{S}}\right)$ and 1,2-bis $(4-$ pyridyl)ethene $\left(L_{L}\right)\left(R h_{2} L_{L}\right) N, N_{0}$-bidentate pyrazine ligands. ${ }^{69}$ The $\beta$ value at the reported $V$ is shown in the legend, and two $\beta$ values are reported for molecules that undergo a transition in the mechanism of CT as a function of $d$. For porphyrin-Cyt-C, the $T_{\mathrm{T}}$ is reported instead of the $\beta .{ }^{17} \mathrm{~A}$ correction factor for effective electrical contact area of $10^{4}$ is taken from ref. 58 and is applied to EGaln junctions from ref. 15 and 60; the solid black arrows link the uncorrected (blackcoloured) and corrected (red-coloured) datasets. The opensource software WebPlotDigitizer (link: https://automeris.io/WebPlotDigitizer/index.html) was utilised to extract numerical data from graphs of the respective publications where needed.

junctions are thermally activated with $E_{\mathrm{a}}=82 \pm 17 \mathrm{mV}$ and $61 \pm 3 \mathrm{mV}$ at $-0.8 \mathrm{~V}$, in both the low and the high conduction states, respectively. Our data suggest that the phase transition in the iron oxide NPs triggers a change in CT from incoherent to coherent tunnelling. The arrows in Fig. $5 \mathrm{~A}$ and $\mathrm{B}$ indicate a sudden change in the slope of the $J(V)$ curve at high $T$ (with a blue arrow that identifies the 'cusp') which disappears at low $T$ accompanied by an increase of the tunnelling current which indicates that a different, more efficient coherent tunnelling pathway is available at low $T$.

\section{Comparison of E2-LFtn with other types of proteins and molecular wires}

Fig. 6 shows how the $J$ values of the junctions with E2-LFtn compares to other types of molecular junctions with biomolecules, saturated alkanethiolate SAMs and long conjugated molecular wires to visualise the efficiency of the CT process across the E2-LFtn. Considering the relatively low $\beta$ of $0.39 \mathrm{~nm}^{-1}$ and relatively high $J$ values, which are considerably greater than that of other proteins (including PS-1, ferritin Aftn-AA, bR and Cyt-C), we observe that E2-LFtn is a highly conductive protein. By comparing the CT rates for E2-LFtn with other molecules with comparable $d$, we note that only oligo-tetrathiafulvalenepyromellitic-diimideimine (OPTI) ${ }^{59}$ junctions support higher CT rates. Fig. 6 also includes our previously reported data on junctions with ferritin which are characterised by two distinct coherent tunnelling regimes with values of $\beta=1.30 \mathrm{~nm}^{-1}$ for $500 \mathrm{Fe}$ to $3000 \mathrm{Fe}$ loading, and $\beta=0.28 \mathrm{~nm}^{-1}$ for $3000 \mathrm{Fe}$ to $4800 \mathrm{Fe}$ loading, ${ }^{15}$ whilst for E2-LFtn only thermally activated regime is observed (see Fig. 4B). Based on our current study and results reported by others, we conclude that Fe ion binding proteins are highly conductive class of biomolecules.

\section{Conclusions}

We demonstrated a transition in the mechanism of CT from incoherent to coherent tunnelling across $\sim 17.7 \mathrm{~nm}$ in bioengineered E2-LFtn based biomolecular tunnel junctions. The investigation of the CT across the E2-LFtn junction as a function of $d_{\mathrm{E} 2-\mathrm{LFtn}}$ and $T$ confirms that the mechanism of the CT is incoherent tunnelling for junctions with apo-E2-LFtn and E2LFtn. In addition, we further report conductance switching in E2-LFtn biomolecular tunnel junctions, and the reversibility of the conductance switching is a function of the Fe ion loading/ cage. Temperature dependent XAS data indicate that this switching is caused by a phase transition in the iron oxide NPs inside E2-LFtn at $T_{\mathrm{T}}=220-240 \mathrm{~K}$. Remarkably, this phase transition changes the mechanism where it transitions from coherent tunnelling at $T<T_{\mathrm{T}}$ to incoherent tunnelling for $T>T_{\mathrm{T}}$ for junctions with 3000Fe loading. In contrast, in 
junctions with $2000 \mathrm{Fe}$ ion loading, the transition is irreversible. Our findings suggest that the iron oxide NPs offer accessible energy states that are separated by a low energy barrier and provide multiple incoherent tunnelling sites resulting in highly conductive CT channels that mediate long-range CT. Our results also highlight the importance of stability as junctions with high Fe ion loadings are more stable than those junctions with low Fe iron loadings. Structural order plays a pivotal role as for protein structures with disorder charge have to hop across segments with high energy barriers while CT across ordered protein structures involves incoherent tunnelling with low energy barriers. The results reported here improve our understanding of the mechanisms of CT across biomolecular junctions. The E2 protein cages are highly conductive, and combined with our previous studies on ferritin, leading us to conclude that iron-binding globular proteins are highly conductive in nature, and can undergo long range coherent activationless CT, and thus, may have potential applications in biomolecular electronic devices.

\section{Conflicts of interest}

There are no conflicts to declare.

\section{Acknowledgements}

The Diamond Light Source and Australian Synchrotron (ANSTO) are acknowledged for assistance in characterisation of the SAMs. D. Q. acknowledges the support of the Australian Research Council (Grant No. FT160100207). We acknowledge the Ministry of Education (MOE) for supporting this research under award no. MOE2019-T2-1-137. Prime Minister's Office, Singapore, under its Medium-sized centre program is also acknowledged for supporting this research. Prof. Jean-Christophe Lacroix are acknowledged for providing us with the CT data from ref.27

\section{References}

1 G. S. Engel, T. R. Calhoun, E. L. Read, T.-K. Ahn, T. Mančal, Y.-C. Cheng, R. E. Blankenship and G. R. Fleming, Nature, 2007, 446, 782-786.

2 E. Collini, C. Y. Wong, K. E. Wilk, P. M. G. Curmi, P. Brumer and G. D. Scholes, Nature, 2010, 463, 644-647.

3 M. L. Ghirardi, A. Dubini, J. Yu and P.-C. Maness, Chem. Soc. Rev., 2009, 38, 52-61.

4 M. A. Grodick, N. B. Muren and J. K. Barton, Biochemistry, 2015, 54, 962-973.

5 A. R. Arnold, M. A. Grodick and J. K. Barton, Cell Chem. Biol., 2016, 23, 183-197.

6 N. Amdursky, D. Ferber, C. A. Bortolotti, D. A. Dolgikh, R. V. Chertkova, I. Pecht, M. Sheves and D. Cahen, Proc. Natl. Acad. Sci. U. S. A., 2014, 111, 5556-5561.

7 M. P. Ruiz, A. C. Aragonès, N. Camarero, J. G. Vilhena, M. Ortega, L. A. Zotti, R. Pérez, J. C. Cuevas, P. Gorostiza and I. Díez-Pérez, J. Am. Chem. Soc., 2017, 139, 15337-15346.
8 C. Romero-Muñiz, M. Ortega, J. G. Vilhena, I. Diéz-Pérez, J. C. Cuevas, R. Pérez and L. A. Zotti, Biomolecules, 2019, 9, 506.

9 J. Zhao, J. J. Davis, M. S. P. Sansom and A. Hung, J. Am. Chem. Soc., 2004, 126, 5601-5609.

10 O. E. Castañeda Ocampo, P. Gordiichuk, S. Catarci, D. A. Gautier, A. Herrmann and R. C. Chiechi, J. Am. Chem. Soc., 2015, 137, 8419-8427.

11 M. Ortega, J. G. Vilhena, L. A. Zotti, I. Díez-Pérez, J. C. Cuevas and R. Pérez, Biomolecules, 2019, 9, 611.

12 N. Amdursky, L. Sepunaru, S. Raichlin, I. Pecht, M. Sheves and D. Cahen, Adv. Sci., 2015, 2, 1400026.

13 L. Sepunaru, N. Friedman, I. Pecht, M. Sheves and D. Cahen, J. Am. Chem. Soc., 2012, 134, 4169-4176.

14 S. Mukhopadhyay, S. Dutta, I. Pecht, M. Sheves and D. Cahen, J. Am. Chem. Soc., 2015, 137, 11226-11229.

15 K. S. Kumar, R. R. Pasula, S. Lim and C. A. Nijhuis, Adv. Mater., 2016, 28, 1824-1830.

16 B. Zhang, W. Song, J. Brown, R. Nemanich and S. Lindsay, J. Am. Chem. Soc., 2020, 142, 6432-6438.

17 N. Amdursky, I. Pecht, M. Sheves and D. Cahen, J. Am. Chem. Soc., 2013, 135, 6300-6306.

18 C. Romero-Muñiz, M. Ortega, J. G. Vilhena, I. Díez-Pérez, J. C. Cuevas, R. Pérez and L. A. Zotti, Phys. Chem. Chem. Phys., 2018, 20, 30392-30402.

19 Y. Li, J. M. Artés, B. Demir, S. Gokce, H. M. Mohammad, M. Alangari, M. P. Anantram, E. E. Oren and J. Hihath, Nat. Nanotechnol., 2018, 13, 1167-1173.

20 J. M. A. Vivancos, J. Hihath and I. Díez-Pérez, Molecular Electronics: An Experimental and Theoretical Approach, Pan Stanford Publishing Pte. Ltd, 2016, pp. 281-323.

21 L. Sepunaru, I. Pecht, M. Sheves and D. Cahen, J. Am. Chem. Soc., 2011, 133, 2421-2423.

22 T. Peng, D. Paramelle, B. Sana, C. F. Lee and S. Lim, Small, 2014, 10, 3131-3138.

23 N. Amdursky, D. Marchak, L. Sepunaru, I. Pecht, M. Sheves and D. Cahen, Adv. Mater., 2014, 26, 7142-7161.

24 S. K. Karuppannan, E. H. L. Neoh, A. Vilan and C. A. Nijhuis, J. Am. Chem. Soc., 2020, 142, 3513-3524.

25 D. Xiang, X. Wang, C. Jia, T. Lee and X. Guo, Chem. Rev., 2016, 116, 4318-4440.

26 L. Luo, S. H. Choi and C. D. Frisbie, Chem. Mater., 2011, 23, 631-645.

27 Q. Van Nguyen, P. Martin, D. Frath, M. L. Della Rocca, F. Lafolet, S. Bellinck, P. Lafarge and J.-C. Lacroix, J. Am. Chem. Soc., 2018, 140, 10131-10134.

28 H. Yan, A. J. Bergren, R. McCreery, M. L. Della Rocca, P. Martin, P. Lafarge and J. C. Lacroix, Proc. Natl. Acad. Sci. U. S. A., 2013, 110, 5326-5330.

29 J. R. Winkler and H. B. Gray, J. Am. Chem. Soc., 2014, 136, 2930-2939.

30 Y. Zhang, C. Liu, A. Balaeff, S. S. Skourtis and D. N. Beratan, Proc. Natl. Acad. Sci. U. S. A., 2014, 111, 10049-10054.

31 C. Joachim and M. A. Ratner, Proc. Natl. Acad. Sci. U. S. A., 2005, 102, 8801-8808. 
32 K. Michaeli, D. N. Beratan, D. H. Waldeck and R. Naaman, Proc. Natl. Acad. Sci. U. S. A., 2019, 116, 5931-5936.

33 C. D. Bostick, S. Mukhopadhyay, I. Pecht, M. Sheves, D. Cahen and D. Lederman, Rep. Prog. Phys., 2018, 81, 026601.

34 M. Buttiker, IBM J. Res. Dev., 1988, 32, 63-75.

35 S. Ho Choi, B. Kim and C. D. Frisbie, Science, 2008, 320, 1482-1486.

36 T. Peng, H. Lee and S. Lim, Biomacromolecules, 2012, 13, 699-705.

37 T. Tosha, M. R. Hasan and E. C. Theil, Proc. Natl. Acad. Sci. U. S. A., 2008, 105, 18182-18187.

38 G. Jutz, P. van Rijn, B. Santos Miranda and A. Böker, Chem. Rev., 2015, 115, 1653-1701.

39 R. M. Cornell and U. Schwertmann, The Iron Oxides, WileyVCH Verlag GmbH \& Co. KGaA, Weinheim, FRG, 2003.

40 J.-P. Jolivet, C. Chanéac and E. Tronc, Chem. Commun., 2004, 477-483.

41 N. Gálvez, B. Fernández, P. Sánchez, R. Cuesta, M. Ceolín, M. Clemente-León, S. Trasobares, M. López-Haro, J. J. Calvino, O. Stéphan, J. M. Domínguez-Vera, N. Gálvez, B. Fernández, P. Sánchez, R. Cuesta, M. Ceolín, M. Clemente-León, S. Trasobares, M. López-Haro, J. J. Calvino, O. Stéphan and J. M. Domínguez-Vera, J. Am. Chem. Soc., 2008, 130, 8062-8068.

42 M. G. Walls, C. Cao, K. Yu-Zhang, J. Li, R. Che and Y. Pan, Microsc. Microanal., 2013, 19, 835-841.

43 D. D. Boland, R. N. Collins, C. J. Miller, C. J. Glover and T. D. Waite, Environ. Sci. Technol., 2014, 48, 5477-5485.

44 S. Das, M. J. Hendry and J. Essilfie-Dughan, Environ. Sci. Technol., 2011, 45, 268-275.

45 L. Yang, C. I. Steefel, M. A. Marcus and J. R. Bargar, Environ. Sci. Technol., 2010, 44, 5469-5475.

46 C. Liu, Z. Zhu, F. Li, T. Liu, C. Liao, J. J. Lee, K. Shih, L. Tao and Y. Wu, Chem. Geol., 2016, 444, 110-119.

47 H. Liu, P. Li, B. Lu, Y. Wei and Y. Sun, J. Solid State Chem., 2009, 182, 1767-1771.

48 X. Liu, W. Jin and E. C. Theil, Proc. Natl. Acad. Sci. U. S. A., 2003, 100, 3653-3658.

49 L. Yuan, L. Jiang, B. Zhang and C. A. Nijhuis, Angew. Chem., Int. Ed., 2014, 53, 3377-3381.

50 S. K. Karuppannan, H. Hongting, C. Troadec, A. Vilan and C. A. Nijhuis, Adv. Funct. Mater., 2019, 1904452, 1904452.

51 J. Zhang, C. Cui and X. Zhou, Chinese Sci. Bull., 2009, 54, $723-726$.
52 T. Rakshit, S. Banerjee, S. Mishra and R. Mukhopadhyay, Langmuir, 2013, 29, 12511-12519.

53 E. Stavitski and F. M. F. de Groot, Micron, 2010, 41, 687-694.

54 M. Koralewski, L. Balejčíková, Z. Mitróová, M. Pochylski, M. Baranowski and P. Kopčanský, ACS Appl. Mater. Interfaces, 2018, 10, 7777-7787.

55 Y. H. Pan, K. Sader, J. J. Powell, A. Bleloch, M. Gass, J. Trinick, A. Warley, A. Li, R. Brydson and A. Brown, J. Struct. Biol., 2009, 166, 22-31.

56 K. Asadi, A. J. Kronemeijer, T. Cramer, L. Jan Anton Koster, P. W. M. Blom and D. M. de Leeuw, Nat. Commun., 2013, 4, 1710.

57 X. Chen, T. Salim, Z. Zhang, X. Yu, I. Volkova and C. A. Nijhuis, ACS Appl. Mater. Interfaces, 2020, 12, 45111-45121.

58 S. Mukhopadhyay, S. K. Karuppannan, C. Guo, J. A. Fereiro, A. Bergren, V. Mukundan, X. Qiu, O. E. Castañeda Ocampo, X. Chen, R. C. Chiechi, R. McCreery, I. Pecht, M. Sheves, R. R. Pasula, S. Lim, C. A. Nijhuis, A. Vilan and D. Cahen, iscience, 2020, 23, 101099.

59 C. E. Smith, S. O. Odoh, S. Ghosh, L. Gagliardi, C. J. Cramer and C. D. Frisbie, J. Am. Chem. Soc., 2015, 137, 15732-15741.

60 L. Jiang, C. S. S. Sangeeth, L. Yuan, D. Thompson and C. A. Nijhuis, Nano Lett., 2015, 15, 6643-6649.

61 S. Raichlin, I. Pecht, M. Sheves and D. Cahen, Angew. Chem., Int. Ed., 2015, 54, 12379-12383.

62 S. H. Choi and C. D. Frisbie, J. Am. Chem. Soc., 2010, 132, 16191-16201.

63 S. H. Choi, C. Risko, M. C. R. Delgado, B. Kim, J.-L. Brédas and C. D. Frisbie, J. Am. Chem. Soc., 2010, 132, 4358-4368.

64 Q. van Nguyen, U. Tefashe, P. Martin, M. L. Della Rocca, F. Lafolet, P. Lafarge, R. L. McCreery and J. C. Lacroix, Adv. Electron. Mater., 2020, 6, 1-11.

65 L. Luo and C. D. Frisbie, J. Am. Chem. Soc., 2010, 132, 8854-8855.

66 A. Morteza Najarian and R. L. McCreery, ACS Nano, 2017, 11, 3542-3552.

67 L. Luo, A. Benameur, P. Brignou, S. H. Choi, S. Rigaut and C. D. Frisbie, J. Phys. Chem. C, 2011, 115, 19955-19961.

68 A. Santos, U. M. Tefashe, R. L. McCreery and P. R. Bueno, Phys. Chem. Chem. Phys., 2020, 22, 10828-10832.

69 D. Bu, Y. Xiong, Y. N. Tan, M. Meng, P. J. Low, D.-B. Kuang and C. Y. Liu, Chem. Sci., 2018, 9, 3438-3450. 